\title{
Achieving Submicrometer Spatial Resolution for Neutron Imaging With Nuclear Emulsion
}

\author{
Abdul Muneem ( $\nabla$ abdul.muneem@riken.jp ) \\ RIKEN \\ Junya Yoshida \\ RIKEN \\ Hiroyuki Ekawa \\ RIKEN \\ Masahiro Hino \\ Kyoto University \\ Katsuya Hirota \\ High Energy Accelerator Research Organization \\ Go Ichikawa \\ High Energy Accelerator Research Organization \\ Ayumi Kasagi \\ RIKEN \\ Masaaki Kitaguchi \\ Nagoya University \\ Naoto Muto \\ Nagoya University \\ Kenji Mishima \\ High Energy Accelerator Research Organization \\ Jameel Nabi \\ University of Wah \\ Manami Nakagawa \\ RIKEN \\ Naotaka Naganawa \\ Nagoya University \\ Takehiko Saito \\ RIKEN
}

\section{Research Article}

Keywords: submicrometer, neutron, nuclear emulsion 
Posted Date: October 21st, 2021

DOI: https://doi.org/10.21203/rs.3.rs-966133/v1

License: (c) (i) This work is licensed under a Creative Commons Attribution 4.0 International License. Read Full License 
particles. They were used to make the first observations of pion ${ }^{16}$, hypernuclei ${ }^{17,18}$, charm particles $^{19}$, and double strangeness hypernuclei ${ }^{20}$ until 1970s. The analysis speed of nuclear emulsions has increased remarkably with improvements in image processing techniques ${ }^{21,22}$. These improvements enable the use of nuclear emulsion in modern experiments to study tau neutrino interactions ${ }^{23}$, direct observation of neutrino oscillations ${ }^{24}$, hypernuclei ${ }^{22,25-28}$, and for several other applications including muon radiography ${ }^{29,30}$.

Since the last decade, it has become possible to produce dedicated nuclear emulsions for specific purposes by controlling the grain size of the silver halide crystals and chemical components to achieve optimal spatial resolution and sensitivity. Fine-grained nuclear emulsions with silver halide crystals having a diameter of less than $0.1 \mu$ m have been developed $^{31-33}$ to detect the tracks of the nuclei recoiled by the so-called weakly interacting massive particles. The sensitivity of the nuclear emulsion was optimised to track the nuclei but was maintained sufficiently low for electrons and $\gamma$-rays ${ }^{34}$. This type of fine-grained nuclear emulsion can be used for neutron imaging by combining it with the ${ }^{10} \mathrm{~B}_{4} \mathrm{C}$ thin layer, which absorbs neutrons and emits charged particles heading in the opposite direction via the following process: ${ }^{10} \mathrm{~B}+\mathrm{n} \rightarrow{ }^{7} \mathrm{Li}+{ }^{4} \mathrm{He}$. When a charged particle passes through an emulsion layer, latent image specks are created in some silver halide crystals along its trajectory. After chemical development, each crystal which contain latent image specks changes to an enlarged silver grain. A series of these grains indicates the tracks of the charged particles.

Naganawa et al. reported that the spatial resolution of a neutron absorption point is less than $0.1 \mu \mathrm{m}$ by using a fine-grained nuclear emulsion under the condition that the track density within ten tracks per $(100 \mu \mathrm{m})^{2}$ in Refs. ${ }^{35}$. However, to apply this detector to neutron imaging, the accumulated track density must be several orders of magnitude higher than the track density. For example, while determining the neutron transmission rate of an area of $(10 \mu \mathrm{m})^{2}$ at an accuracy of $1 \%$ compared to the surrounding area, the magnitude of the track density should be $10^{6}$ per $(100 \mu \mathrm{m})^{2}$ to reduce the statistical error. The recorded tracks get severely overlapped at such a high track density condition. Hence, it becomes difficult to use an analysis method for individual tracks. Hirota et al. attempted neutron imaging under a high track density condition using fine-grained nuclear emulsion $^{36}$. They succeeded in visualizing the inner components of a crystal oscillator chip, for example, gold wires with a diameter of approximately $30 \mu \mathrm{m}$. However, the spatial resolution was not evaluated quantitatively. Therefore, it is important to conduct a quantitative analysis of the spatial resolution of this neutron imaging technique with an object having well-defined micrometer-scale structure.

In this study, we developed a new technique for neutron imaging using a fine-grained nuclear emulsion. We attempted neutron imaging using the developed technique for the Siemens star test pattern and the gadolinium-based grating with a periodic structure of $9 \mu \mathrm{m}$. The spatial resolution of neutron imaging was quantitatively deduced using the gadolinium-based grating.

a

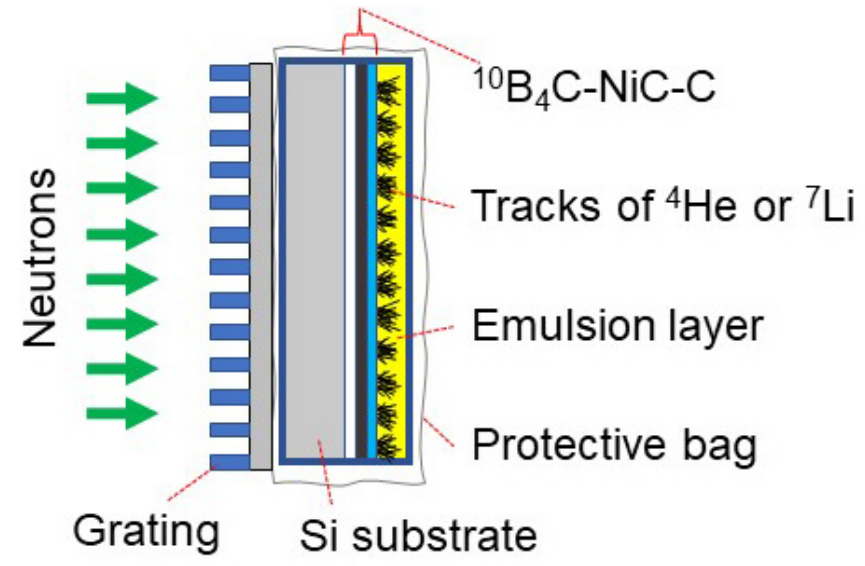

b

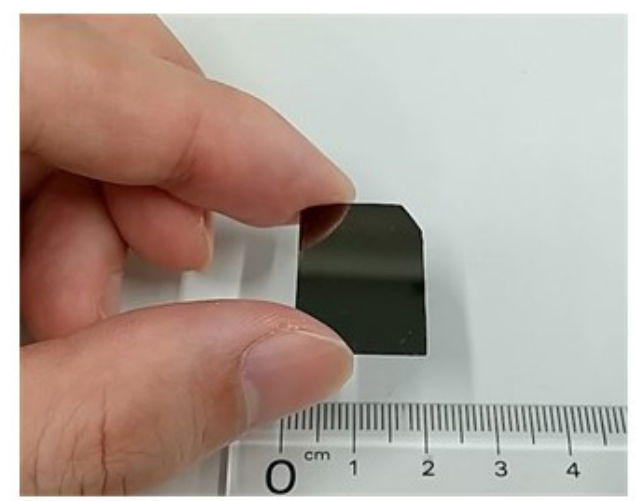

Figure 1. Developed neutron detector with the nuclear emulsion. a Schematic layout of the neutron detector using a fine-grained emulsion packed in a protective bag combined with a gadolinium-based grating. b Photograph of developed neutron detector. The size of the detector was $25 \mathrm{~mm} \times 15 \mathrm{~mm}$.

\section{Results}

\section{Neutron detector system using a fine-grained nuclear emulsion} silicon substrate. On this substrate, ${ }^{10} \mathrm{~B}_{4} \mathrm{C}, \mathrm{NiC}$, and C layers of thickness $0.23 \mu \mathrm{m}, 46 \mathrm{~nm}$, and $14 \mathrm{~nm}$, respectively, were 

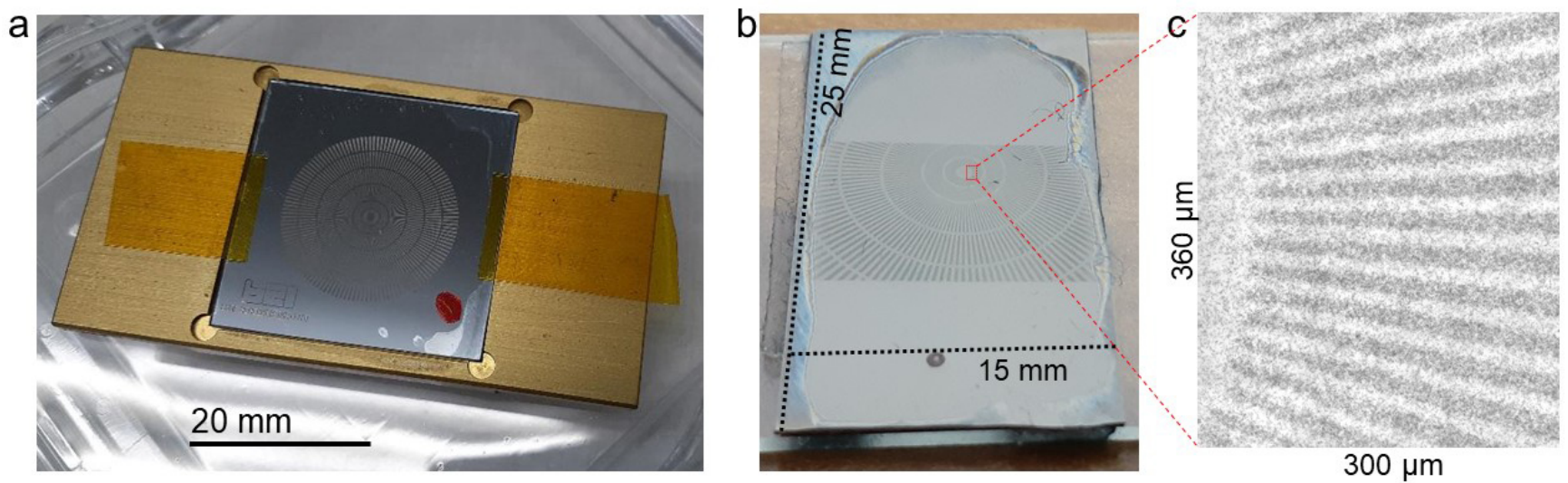

Figure 2. Neutron imaging of the Siemens star test pattern. a Photograph of the Siemens star test pattern. It consists of a thin layer of gadolinium on a quartz substrate and is housed in an aluminum frame. $\mathbf{b}$ Photograph of the developed detector irradiated with the neutron beam through the pattern. The pattern is visible on the emulsion layer to the eyes. $\mathbf{c}$ Micrograph of the recorded image pattern in the nuclear emulsion, showing the innermost edge of the pattern. The sizes of the vertical and horizontal directions of the figure correspond to 360 and $300 \mu \mathrm{m}$, respectively, as shown in the figure.

formed by using an ion beam sputtering technique ${ }^{37}$. The ${ }^{10} \mathrm{~B}_{4} \mathrm{C}$ layer converts an incident neutron into two charged particles, ${ }^{4} \mathrm{He}$ and ${ }^{7} \mathrm{Li}$. The $\mathrm{NiC}$ layer is used to physically stabilise the ${ }^{10} \mathrm{~B}_{4} \mathrm{C}$ layer. The $\mathrm{C}$ layer was used for chemical protection from the $\mathrm{NiC}$ layer and for providing a strong adhesion to the emulsion. An emulsion layer of thickness of $10 \mu \mathrm{m}$ was formed on the sputtered layers. It is composed of silver halide crystals of approximately $40 \mathrm{~nm}$ diameter dispersed in gelatin and including polyvinyl alcohol.

During the beam exposure, the neutron detector, and the grating were placed close together to minimise the blurring effects induced by the spreading of the transmitted neutron beam, as shown in Figure 1a. A portion of the incident neutrons is absorbed in the imaging subject, and some of the remaining neutrons are absorbed in the ${ }^{10} \mathrm{~B}_{4} \mathrm{C}$ converter layer. When a ${ }^{10} \mathrm{~B}$ nucleus absorbs a neutron, the reaction products, ${ }^{4} \mathrm{He}$ and ${ }^{7} \mathrm{Li}$ that are emitted head off in the opposite direction. One of them passes through the emulsion layer, creating a track of a few micrometers in length. Figure $1 \mathrm{~b}$ shows a photograph of the developed detector. After the chemical development, grayscale images are obtained with submicrometer precision from the optical microscope, and silver grains become visible.

\section{Imaging of the PSI Siemens star test pattern}

Figure 2a shows a photograph of the Siemens star test pattern used in the experiments performed in this study. This pattern is designed by a group based at the Paul Scherrer Institute (PSI), Villigen, Switzerland. A prototype of the test pattern is described in Ref. ${ }^{38}$. The pattern consists of 128 spokes in a circular area of $20 \mathrm{~mm}$ diameter and is made of a thin layer (in the order of 5 $\mu \mathrm{m}$ ) of gadolinium on a quartz wafer with a thickness of $0.7 \mathrm{~mm}$. The space between the spokes becomes narrower towards the center of the circle. The innermost spoke has a period of $10 \mu \mathrm{m}$, which corresponds to 100 line pairs per millimeter. Then, the spatial resolution of an imaging device is assessed by how fine the intervals of the spokes can be resolved.

Figure $2 \mathrm{~b}$ shows a photograph of the developed detector irradiated with the neutron beam through the pattern. The pattern transferred on the emulsion layer is visible to the naked eyes even before being observed under optical microscope. The dark regions correspond to sites where silver grains have been produced by the emitted ${ }^{4} \mathrm{He}$ or ${ }^{7} \mathrm{Li}$ during the neutron absorption events. Other regions correspond to sites where the neutrons are absorbed by gadolinium on the pattern, and neutron absorption events in the ${ }^{10} \mathrm{~B}_{4} \mathrm{C}$ layer are suppressed. Figure $2 \mathrm{c}$ shows a micrograph of the nuclear emulsion, which recorded the thinnest spokes near the center of the pattern. Because the innermost spokes having a period of $10 \mu \mathrm{m}$ are clearly resolved, this result shows the ability of the fine-grained nuclear emulsion to achieve the spatial resolution of micrometer-scale or better.

\section{Imaging of the gadolinium-based grating}

The spatial resolution of the neutron imaging with the detector developed in the present work was investigated by using a grating made of gadolinium with a periodic structure of $9 \mu \mathrm{m}$, that is, 111 line pairs per millimeter. Figure 3a shows an image of the gadolinium-based grating formed on the silicon substrate. The grating was initially made for a Talbot-Lau interferometer for neutron phase imaging ${ }^{39}$. Figure $3 \mathrm{~b}$ shows a scanning electron microscopy (SEM) image of the grating produced by the same process as that used in the performed experiments. Figure 3c shows a SEM image of the cross-section of a gadolinium tooth and Figure $3 \mathrm{~d}$ shows a schematic view of the gadolinium teeth. Gadolinium teeth with a width of approximately $5 \mu \mathrm{m}$ 

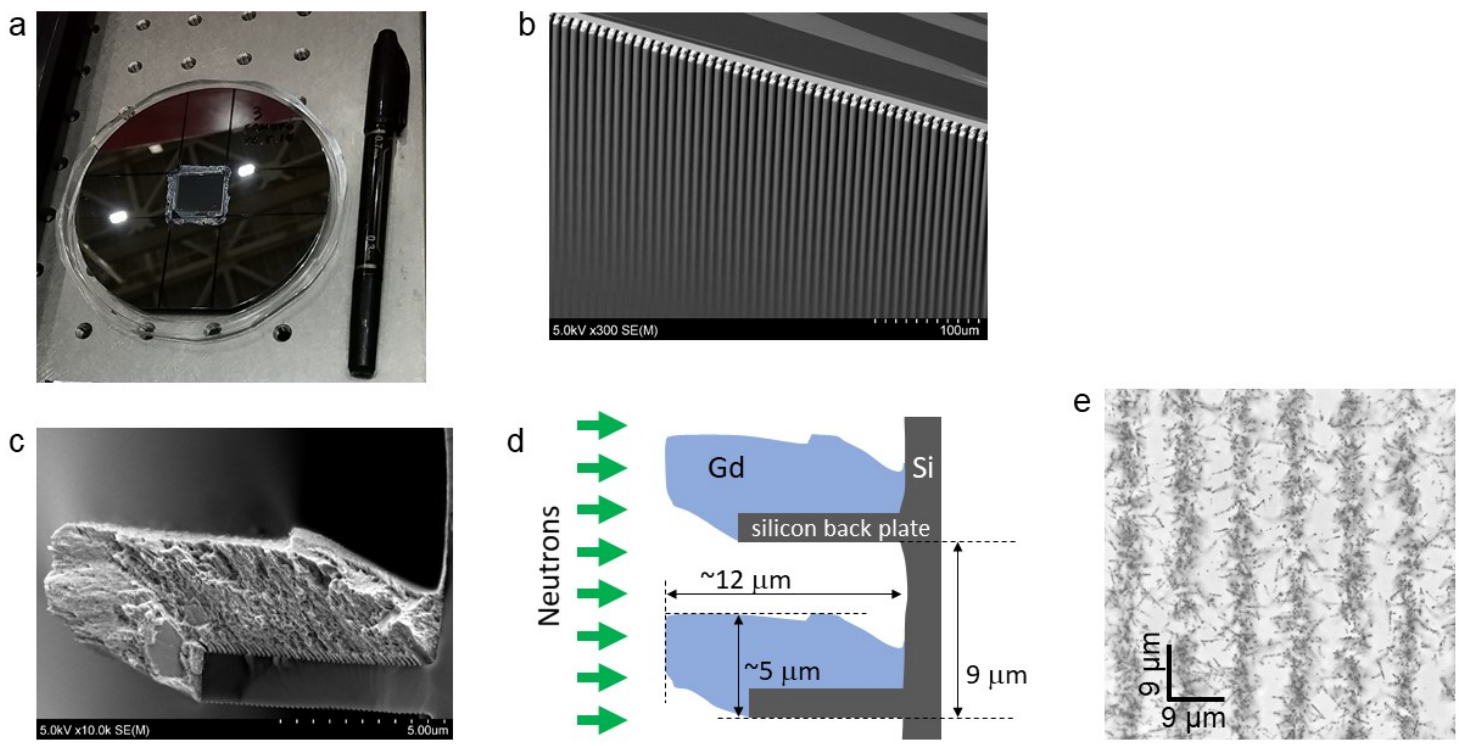

Figure 3. Neutron imaging of the gadolinium-based grating. a A picture of a gadolinium-based grating with dimensions of $20 \mathrm{~mm} \times 20 \mathrm{~mm}$. It was formed on a $525 \mu \mathrm{m}$-thick silicon substrate and mounted at the centre of a $525 \mu \mathrm{m}$-thick five-inch silicon wafer. b Image of the structure of the gadolinium teeth obtained by scanning electron microscopy (SEM). $\mathbf{c}$ SEM image of the cross-section of a single gadolinium tooth. $\mathbf{d}$ Schematic view of the gadolinium teeth. The direction of the irradiated neutrons was from left to right. A portion of the incident neutron is absorbed by the gadolinium, while the remnant neutrons pass through the empty spaces. e Optical micrograph of the neutron detector that recorded the tracks during neutron capture events through the grating. One side of this micrograph corresponds to 1024 pixels, which is equal to $56 \mu \mathrm{m}$.

and empty spaces of $4 \mu \mathrm{m}$ were formed periodically. Each tooth was formed by depositing gadolinium from an oblique angle onto the ridge-like structures formed on the silicon substrate ${ }^{39}$.

Figure $3 \mathrm{e}$ shows a micrograph of tracks recorded in the nuclear emulsion layer. The dimensions and number of pixels of this micrograph are $56 \mu \mathrm{m} \times 56 \mu \mathrm{m}$ and 1024 pixels $\times 1024$ pixels, respectively. The periodic structure with $9 \mu \mathrm{m}$ is clearly visible.

\section{Evaluation of the spatial resolution}

We produced edge profiles from the obtained micrographs of the neutron detector and fitted the Gaussian error function to evaluate the edge rise. The error function is described by the following equation:

$$
\text { Errorfunction }(x)=\frac{A}{\sqrt{2 \pi} \sigma} \int_{0}^{x} \exp \left(-\frac{(t-\mu)^{2}}{2 \sigma^{2}}\right) \mathrm{d} t+B .
$$

where $\mathrm{A}$ is the amplitude, $\mathrm{B}$ is the base value, $\mu$ is the mean, and $\sigma$ is the diffuseness of the edge.

Before the fitting process, we converted the grayscale micrograph into a binarised image, as shown in Figure 4a, using the image processing described in the Methods section. The coordinates are also shown in the figure. In the binarised image, the white and black pixels correspond to the areas where the silver grains are present and absent, respectively. Using the image, the number of the white pixels in the direction parallel to the grating (Y-direction) was calculated at a certain X-value with a range of bin sizes, as shown in Figure 4b. The length of the error bar for each point corresponds to the square root of the number of white pixels in each line. Then, we divided this graph into individual edge sections by detecting the positions of the peaks and valleys of the periodic structure. The green lines in Figure $4 \mathrm{~b}$ represent the defined boundaries of each edge section. The falling and rising edges are treated separately in the subsequent analyses to distinguish between the two sides of the gadolinium tooth, that is, one with a silicon back plate and one without it, as shown in Figure 3d. These two sides correspond to the falling and rising edges, respectively. To exclude the incomplete stripes in the peripheral part of the micrograph and equalise the number of rising and falling edges, we defined pairs of adjacent rising and falling edges and used the paired edges in the subsequent 

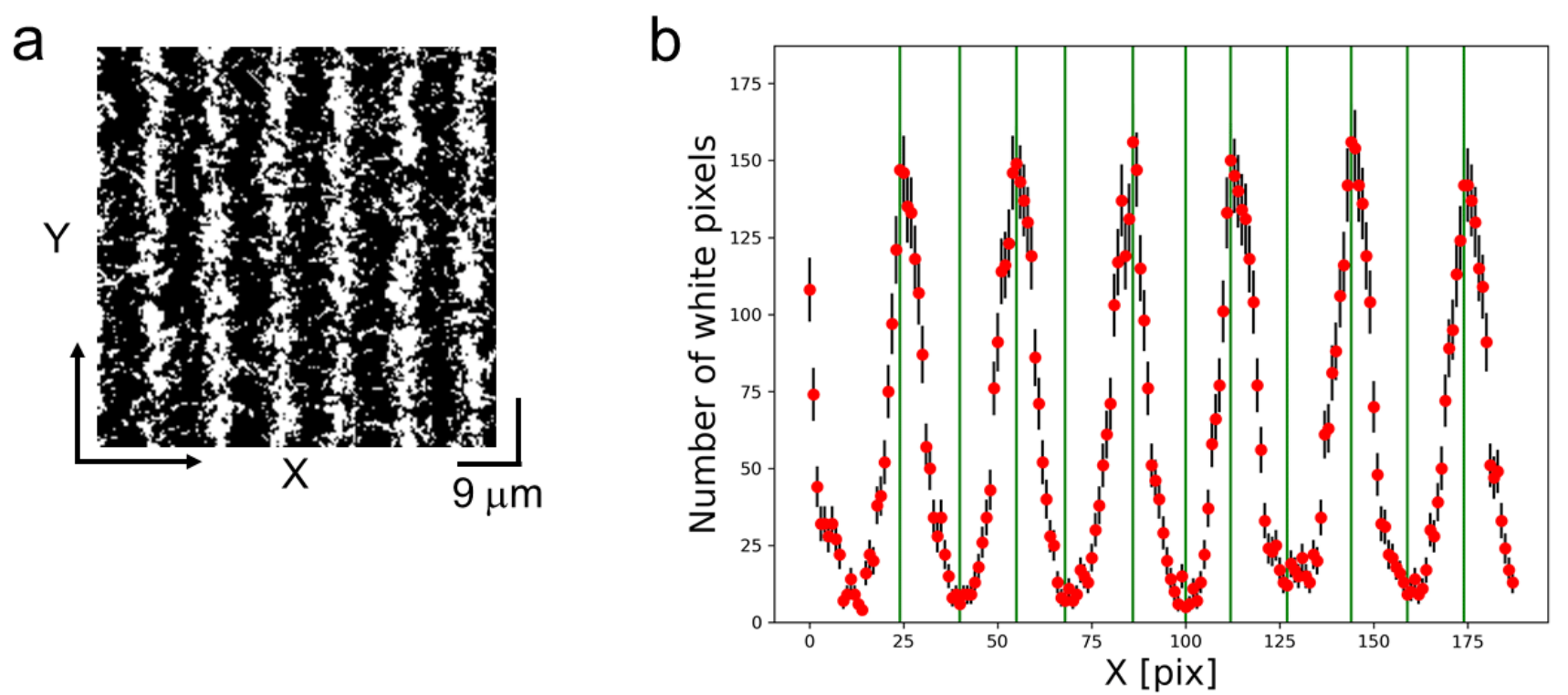

Figure 4. Data processing of neutron imaging of the gadolinium-based grating. a A resised and binarised micrograph of an optical micrograph of the neutron detector, as shown in Figure $3 \mathrm{e}$. The dimensions of this micrograph were $56 \mu \mathrm{m} \times 56 \mu \mathrm{m}$. It was scaled down to 188 pixels $\times 188$ pixels, such that the size of one pixel is equivalent to $0.3 \mu \mathrm{m}$, which is the typical apparent diameter of a silver grain. Each white pixel corresponds to an area in which silver grains were present. b Number of white pixels along as a function of the X position. The length of each error bar corresponds to the square root of the number of white pixels. The green lines are the positions of the peaks and valleys, and the defined boundaries of individual edge sections. To equalise the number of rising and falling edges in the subsequent analysis, we only used the four pairs of rising and four falling edges in the region $(40<X<159)$ from this data.

analysis. We applied this data processing to the obtained micrographs of 168 field-of-views, which correspond to an area of $0.65 \mathrm{~mm}^{2}$.

Figure 5a,b shows the fitting for a rising edge and falling edge, respectively, using Eq. (1). The red dotted lines show the fitted error function curves. The fitting was conducted in the range between the maximum and minimum points in each cut section. A total of 709 rising and 709 falling edges were used in the analysis, and Figure 6 shows the distributions of the reduced $\chi^{2}$ of the fitting.

Because the diffuseness distributions of the rising and falling edges are asymmetric with a tail to the right side, as shown in Figure 7, we employed a log-normal distribution described by the following equation:

$$
\text { Log-normaldistribution }(x)=\frac{A}{\sqrt{2 \pi} \sigma x} \exp \left(-\frac{(\ln x-\mu)^{2}}{2 \sigma^{2}}\right) .
$$

where $\mathrm{A}$ is the amplitude factor, $\exp (\mu)$ is the median value, and $\sigma$ is a parameter that correlates with the width of this distribution. The $\chi^{2}$ divided by the number of degrees of freedom of the fitting for the rising and falling edges were $25.9 / 19$ and $23.73 / 16$, respectively. The deduced median values of the diffuseness distributions of the rising and falling edges are 0.91 \pm 0.01 and $0.88 \pm 0.01 \mu \mathrm{m}$, and their mean values are $0.96 \pm 0.01$ and $0.93 \pm 0.01 \mu \mathrm{m}$, respectively. These values are less than $1 \mu \mathrm{m}$, as shown in Figure 7.

\section{Discussion}

We evaluated the median values of the diffuseness for the rising and falling edges to be $0.91 \pm 0.01$ and $0.88 \pm 0.01 \mu \mathrm{m}$, respectively. These values for the rising and falling edges are consistent within three standard deviations. Here, the shape of the gadolinium teeth is not a perfect rectangle because of the production process, as described in Ref. ${ }^{40,41}$. Therefore, the analysis presented in this paper is an inclusive assessment that includes the effects of the resolution of the detector and the shape of the gadolinium tooth. However, because the median values of the diffuseness are less than $1 \mu \mathrm{m}$, we have achieved a submicrometer spatial resolution using a fine-grained nuclear emulsion for neutron imaging. To the best of our knowledge, the achieved spatial resolution is the best among the neutron imaging devices reported to date. 

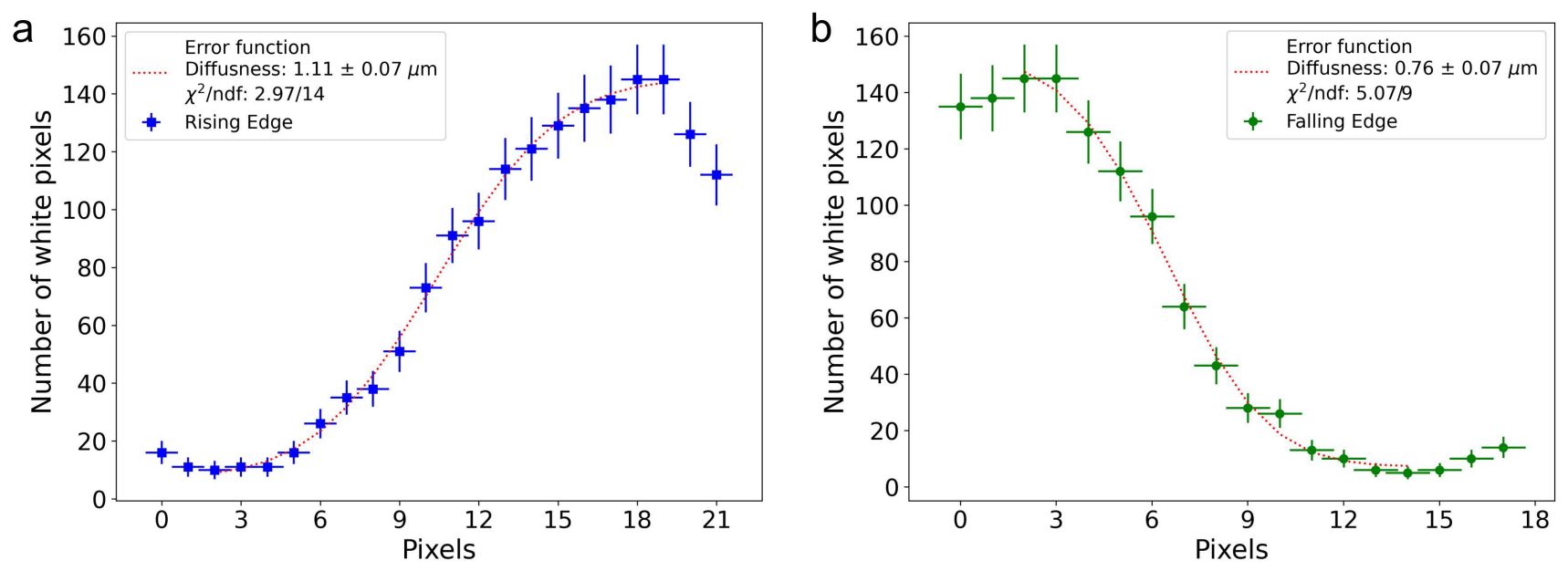

Figure 5. Examples of data points of the edges and their fitting with the Gaussian error function. Panels a and $b$ show the rising and falling edges, respectively. The red dotted lines represent the curves obtained by the fitting. The fitting range is defined between the minimum and maximum points.

As demonstrated herein, precise neutron imaging using fine-grained nuclear emulsion will open a new avenue for nondestructive inspection to visualize the internal structure of advanced materials such as memory chips and fuel cells. For fuel cells, it is essential to visualize the water distribution during operation to improve its performance. Moreover, this technique can be used in the development of neutron optics elements such as gadolinium-based gratings. For instance, gadolinium-based gratings with micrometer-scale structures are used in neutron imaging with Talbot-Lau interferometry, and gratings with finer structures lead to a better wavelength resolution of Talbot-Lau imaging. The use of fine-grained nuclear emulsion detectors in the development of gratings can improve the resolution of interferometry techniques. Another potential application of precise neutron imaging is the inspection of test patterns for neutron imaging with a micrometer-scale structure. As the spatial resolution of neutron imaging approaches the micrometer scale, test patterns with micrometer-scale become important for evaluating imaging quality ${ }^{9}$. It is essential to observe such test patterns with neutron transmission, in addition to scanning electron microscopy and X-ray microscopy. Moreover, this technique could also be helpful in evaluating newly developed neutron imaging devices aiming to achieve a micrometer-scale spatial resolution. Neutron imaging may be conducted for a test pattern with a micrometer-scale structure using the nuclear emulsion and another developing neutron imaging device. The resolution of the developing device and the diffuseness caused by the beam divergence separately can be deduced by analysing and comparing the obtained images.

The fine-grained nuclear emulsion detector is lightweight, thin, small (even adjustable to sample objects with an area of approximately $100 \mathrm{~cm}^{2}$ ), and can be set without an electrical power supply. Therefore, this detector can be added to existing imaging systems without major modifications and can be used as a complementary imaging device. Precise neutron imaging using fine-grained nuclear emulsion may provide new insights in a wide range of research fields and potential applications, for example, biology, geomechanics, and advanced materials such as semiconductor devices, batteries, and fuel cells.

\section{Methods}

\section{Detector}

The ${ }^{10} \mathrm{~B}_{4} \mathrm{C}, \mathrm{NiC}$, and $\mathrm{C}$ layers were formed on a silicon substrate by an ion beam sputtering machine available at the Institute for Integrated Radiation and Nuclear Science of Kyoto University ${ }^{37}$. The thickness of the ${ }^{10} \mathrm{~B}_{4} \mathrm{C}$ layer was adjusted by controlling the sputtering time, using the value of $1.25 \mathrm{~nm} / \mathrm{min}$ of film growth speed measured in a preliminary experiment. The detection efficiency of neutrons with this ${ }^{10} \mathrm{~B}_{4} \mathrm{C}$ layer was measured to be approximately $0.4 \%$ for neutrons with a velocity of $2200 \mathrm{~m} / \mathrm{s}$. These three layers were formed on a 3-inch silicon substrate with a thickness of $0.4 \mathrm{~mm}$. The emulsion layer was formed on a piece of substrate in a dark room by pouring and drying the fine-grained nuclear emulsion. Each detector was packed with a light- and moisture-proof laminated bag, which was composed of nylon, polyethylene, aluminum, polyethylene, and black polyethylene layers having thicknesses of $15 \mu \mathrm{m}, 13 \mu \mathrm{m}, 7 \mu \mathrm{m}, 13 \mu \mathrm{m}$, and $35 \mu \mathrm{m}$, respectively. 


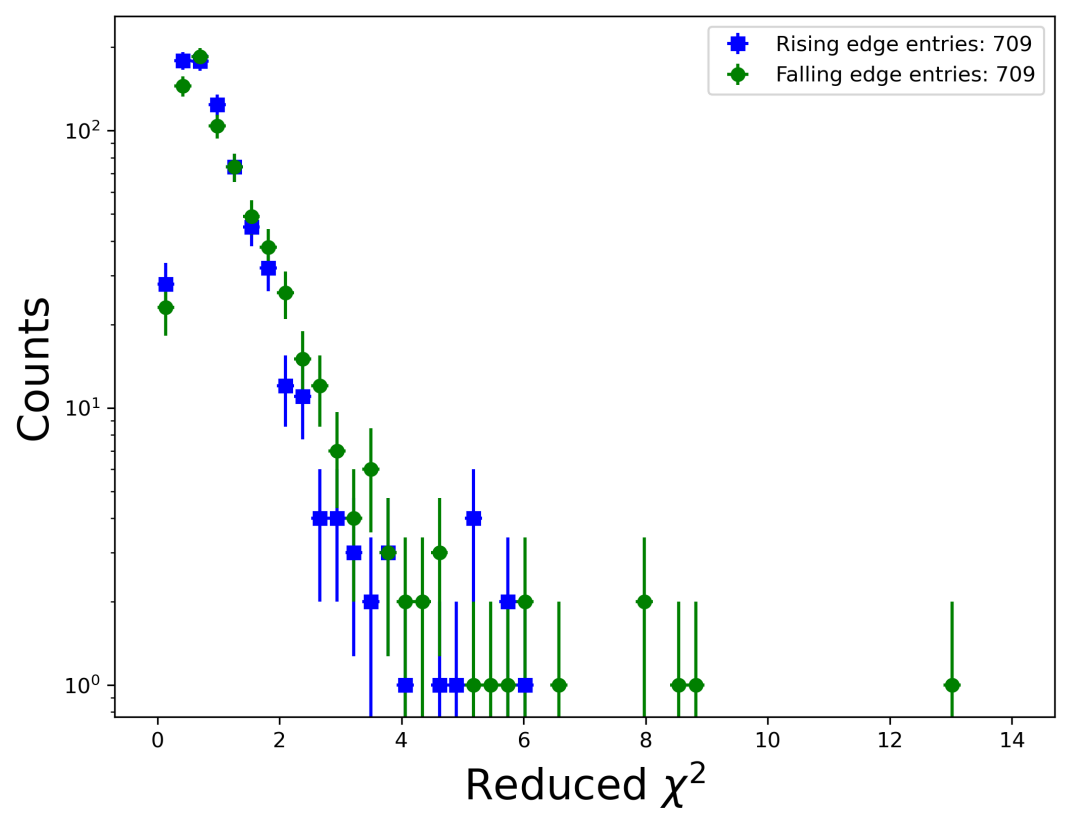

Figure 6. Distribution of reduced $\chi^{2}$ values for the fitting with the error function. A total of 709 rising and 709 falling edges were used.

\section{Beam exposure}

We used the low-divergence beam branch ${ }^{42}$ of BL05 in the Materials and Life Science Experimental Facility (MLF) at the Japan Proton Accelerator Research Complex (J-PARC). This is one of the most suitable beam branches in the MLF for this experiment because it provides a reasonably intense neutron beam owing to a coupled neutron moderator.

For neutron imaging of the PSI Siemens star, the divergences of the beam in the horizontal and vertical directions were set to $0.3 \mathrm{mrad}$ and $1.0 \mathrm{mrad}$, respectively. The typical beam power and the neutron flux during the experiment were $0.7 \mathrm{MW}$ and $10^{5} \mathrm{n} / \mathrm{cm}^{2} / \mathrm{s}$, respectively. The irradiation time was $9 \mathrm{~h}$ to accumulate approximately $10^{4}$ tracks per $(100 \mu \mathrm{m})^{2}$. In contrast, for neutron imaging of the gadolinium-based grating, the beam divergence in the vertical direction was set to $10 \mathrm{mrad}$ because the beam divergence parallel to the grating does not affect the blurring of the image. Then, the neutron flux was $2 \times 10^{6} \mathrm{n} / \mathrm{cm}^{2} / \mathrm{s}$, and the neutron irradiation time was $2.8 \mathrm{~h}$ to accumulate approximately $2 \times 10^{4}$ tracks per $(100 \mu \mathrm{m})^{2}$.

\section{Chemical development}

After neutron irradiation, the chemical development of the detectors was conducted in a dark room. The development process involves immersing the detectors in the developing, stopping, and fixing solutions. The main agent of the developer was ascorbic acid, and the temperature was fixed to $20^{\circ} \mathrm{C}$. The immersion times in the developing, stopping, and fixing solutions were $5 \mathrm{~min}, 5 \mathrm{~min}$, and $12 \mathrm{~min}$, respectively. The detectors were then rinsed in tap water for $10 \mathrm{~min}$ and dried for $1 \mathrm{~h}$.

\section{Scanning of the neutron detector}

A dedicated optical microscope with an epi-illumination system was used to scan the emulsion layer on a non-transparent silicon substrate. The light source of this system was a $1 \mathrm{~W}$ LED with a peak wavelength of $455 \mathrm{~nm}$. The objective lens is an oil-immersion type with a magnification of 100x with a numerical aperture (NA) of 1.45 (Nikon Plan Apo). The depth of field was derived as $2 \times \lambda / \mathrm{NA}^{2} \simeq 0.43 \mu \mathrm{m}$. The micrographs were acquired as 8-bit grayscale images with a complementary metal-oxide-semiconductor (CMOS) image sensor with 2048 pixels $\times 2048$ pixels (SENTECH CMB401PCL) at speeds of up to 160 frames per second. The length of one side of the field of view is approximately $112 \mu \mathrm{m}$, that is, a single pixel corresponds to $0.055 \mu \mathrm{m}$. We only use the central region of the micrograph (i.e., 1024 pixels $\times 1024$ pixels), because optical aberration in the periphery of the field of view reduces the quality of the image. Three independent stepping motors control the stage of the microscope along the two horizontal axes and the vertical axis. This microscope system acquires sequential images along the vertical axis with a pitch of $0.3 \mu \mathrm{m}$ from the upper boundary to the lower boundary of the emulsion layer. The system shifts the field of view in the horizontal direction at a $50 \mu \mathrm{m}$ pitch and scans the specified area. 


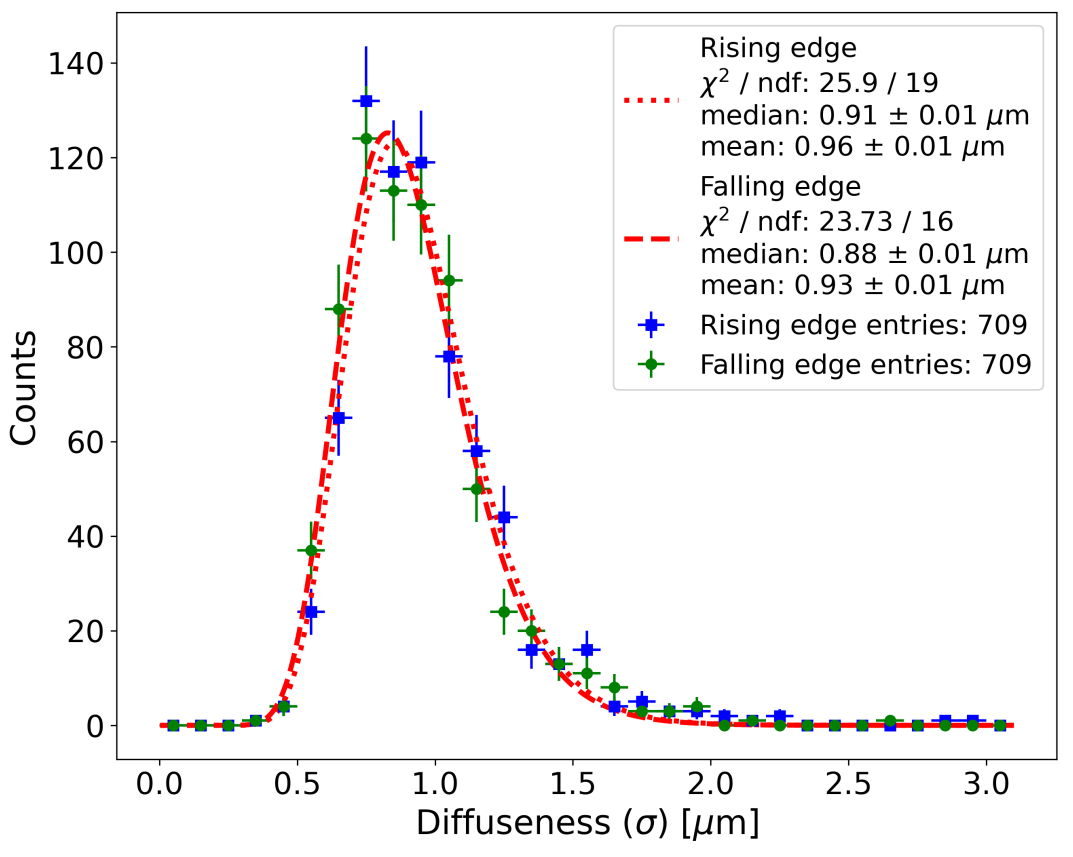

Figure 7. Distributions of the diffuseness and their fitting results. The blue and green marks represent the obtained diffuseness distributions for the rising and falling edges, respectively. The dotted and dashed red lines are the log-normal fit curves for the rising and falling edges, respectively.

\section{Image processing}

The following image processing sequence was carried out before evaluating the spatial resolution of the neutron imaging: creation of division images, resising, and binarization. The images acquired by the optical microscope contain shades of dust on the image sensor and non-uniformity of brightness due to optical conditions. To remove them, the division images were created by the following process. We calculated the average brightness for each pixel and generated a mean image using a set of sequential tomographic images acquired at different depths of the focus plane. The image at the depth of the boundary between the sputtered $\mathrm{C}$ layer and the emulsion layer was selected. The brightness value of each image was divided by the brightness value of the corresponding pixel in the mean image. The division images were resised to 188 pixels $\times 188$ pixels such that the size of one pixel was equal to $0.3 \mu \mathrm{m}$, corresponding to the typical apparent diameter of the silver grain. Furthermore, we applied Otsu's binarization ${ }^{43}$ to convert the grayscale images to binarised images by employing a non-parametric and unsupervised method, as shown in Figure 4a. Here, white and black pixels correspond to areas where the silver grains are present and absent, respectively.

\section{Data availability}

The data that support the findings of this study are available from the corresponding author upon reasonable request.

\section{Code availability}

Computer codes that support the findings of this study are available from the corresponding author upon reasonable request.

\section{References}

1. Strobl, M. et al. Topical review: Advances in neutron radiography and tomography. JOURNAL OF PHYSICS D: APPLIED PHYSICS 42, 243001, DOI: https://doi.org/10.1088/0022-3727/42/24/243001 (2009).

2. Hussey, D. S. \& Jacobson, D. L. Applications of neutron imaging and future possibilities. Neutron News 26, 19-22, DOI: https://doi.org/10.1080/10448632.2015.1028273 (2015).

3. Lehmann, E. H., Boillat, P., Kaestner, A., Vontobel, P. \& Mannes, D. Neutron imaging methods for the investigation of energy related materials. EPJ Web Conf. 104, 01007, DOI: https://doi.org/10.1051/epjconf/201510401007 (2015). 
4. Kardjilov, N., Manke, I., Woracek, R., Hilger, A. \& Banhart, J. Advances in neutron imaging. Mater. Today 21, 652-672, DOI: https://doi.org/10.1016/j.mattod.2018.03.001 (2018).

5. Tengattini, A., Lenoir, N., Andò, E. \& Viggiani, G. Neutron imaging for geomechanics: A review. Geomech. for Energy Environ. 27, 100206, DOI: https://doi.org/10.1016/j.gete.2020.100206 (2021). Deformation Characteristics of Geomaterials - selected papers for 7th International Symposium, IS-Glasgow2019.

6. Banhar, J. Advanced tomographic methods in materials research and engineering. (Oxford: Oxf. Univ. Press. DOI: https://doi.org/10.1093/acprof:oso/9780199213245.001.0001 ((ed) 2008).

7. Tremsin, A. et al. Improved efficiency of high resolution thermal and cold neutron imaging. NUCL INSTRUM METH PHYS RES A 628, 415-418, DOI: https://doi.org/10.1016/j.nima.2010.07.014 (2011).

8. Hussey, D. et al. A new cold neutron imaging instrument at nist. Phys. Procedia 69, 48-54, DOI: https://doi.org/10.1016/j. phpro.2015.07.006 (2015).

9. Trtik, P. et al. Improving the spatial resolution of neutron imaging at paul scherrer institut - the neutron microscope project. Phys. Procedia 69, 169-176, DOI: https://doi.org/10.1016/j.phpro.2015.07.024 (2015).

10. Trtik, P. \& Lehmann, E. H. Progress in high-resolution neutron imaging at the paul scherrer institut - the neutron microscope project. J. Physics: Conf. Ser. 746, 012004, DOI: https://doi.org/10.1088/1742-6596/746/1/012004 (2016).

11. Bingham, P., Santos-Villalobos, H., Lavrik, N., Gregor, J. \& Bilheux, H. Magnified neutron radiography with coded sources. Phys. Procedia 69, 218-226, DOI: https://doi.org/10.1016/j.phpro.2015.07.031 (2015).

12. Morgano, M. et al. Unlocking high spatial resolution in neutron imaging through an add-on fibre optics taper. Opt. Express 26, 1809-1816, DOI: https://doi.org/10.1364/OE.26.001809 (2018).

13. Trtik, P. et al. PSI 'Neutron Microscope' at ILL-D50 Beamline - First Results. Mater. Res. Proc. 15, 23-28, DOI: https://doi.org/10.21741/9781644900574-4 (2020).

14. Woracek, R. et al. Spatially resolved time-of-flight neutron imaging using a scintillator cmos-camera detector with khz time resolution. Opt. Express 27, 26218-26228, DOI: https://doi.org/10.1364/OE.27.026218 (2019).

15. Hussey, D. S., LaManna, J. M., Baltic, E. \& Jacobson, D. L. Neutron imaging detector with $2 \mu \mathrm{m}$ spatial resolution based on event reconstruction of neutron capture in gadolinium oxysulfide scintillators. Nucl. Instruments Methods Phys. Res. Sect. A: Accel. Spectrometers, Detect. Assoc. Equip. 866, 9-12, DOI: https://doi.org/10.1016/j.nima.2017.05.035 (2017).

16. Lattes, C. M. G., Occhialinidr., G. P. S. \& Powelldr, C. F. Observations on the tracks of slow mesons in photographic emulsions*. Nature 160, 453-456, DOI: https://doi.org/10.1038/160453a0 (1947).

17. Danysz, M. \& Pniewski, J. Delayed disintegration of a heavy nuclear fragment: I. The London, Edinburgh, Dublin Philos. Mag. J. Sci. 44, 348-350, DOI: https://doi.org/10.1080/14786440308520318 (1953).

18. Davis, D. 50 years of hypernuclear physics: I. the early experiments. Nucl. Phys. A 754, 3-13, DOI: https://doi.org/10. 1016/j.nuclphysa.2005.01.002 (2005). Proceedings of the Eighth International Conference on Hypernuclear and Strange Particle Physics.

19. Niu, K., Mikumo, E. \& Maeda, Y. A Possible Decay in Flight of a New Type Particle. Prog. Theor. Phys. 46, 1644-1646, DOI: https://doi.org/10.1143/PTP.46.1644 (1971). https://academic.oup.com/ptp/article-pdf/46/5/1644/5271903/46-5-1644. pdf.

20. Danysz, M. et al. The identification of a double hyperfragment. Nucl. Phys. 49, 121-132, DOI: https://doi.org/10.1016/ 0029-5582(63)90080-4 (1963).

21. Yoshimoto, M., Nakano, T., Komatani, R. \& Kawahara, H. Hyper-track selector nuclear emulsion readout system aimed at scanning an area of one thousand square meters. Prog. Theor. Exp. Phys. 2017, DOI: https://doi.org/10.1093/ptep/ptx131 (2017).

22. Saito, T. R. et al. New directions in hypernuclear physics. Nat. Rev. Phys. DOI: https://doi.org/10.1038/ s42254-021-00371-w (2021).

23. Kodama, K. et al. Final tau-neutrino results from the DONuT experiment. Phys. Rev. D 78, 052002, DOI: https: //doi.org/10.1103/PhysRevD.78.052002 (2008).

24. OPERA Collaboration et al. Observation of tau neutrino appearance in the CNGS beam with the OPERA experiment. Prog. Theor. Exp. Phys. 2014, DOI: https://doi.org/10.1093/ptep/ptu132 (2014). 101C01, https://academic.oup.com/ptep/ article-pdf/2014/10/101C01/4414189/ptu132.pdf. 
25. Hiyama, E. \& Nakazawa, K. Structure of $\mathrm{S}=-2$ Hypernuclei and Hyperon-Hyperon Interactions. Annu. Rev. Nucl. Part. Sci. 68, 131-159, DOI: 10.1146/annurev-nucl-101917-021108 (2018). https://doi.org/10.1146/annurev-nucl-101917-021108.

26. Ekawa, H. et al. Observation of a Be double-Lambda hypernucleus in the J-PARC E07 experiment. Prog. Theor. Exp. Phys. 2019, DOI: https://doi.org/10.1093/ptep/pty149 (2019). 021D02, https://academic.oup.com/ptep/article-pdf/2019/2/ 021D02/27970468/pty149.pdf.

27. Hayakawa, S. H. et al. Observation of Coulomb-Assisted Nuclear Bound State of $\Xi^{-}-{ }^{14}$ N System. Phys. Rev. Lett. 126, 062501, DOI: https://doi.org/10.1103/PhysRevLett.126.062501 (2021).

28. Yoshimoto, M. et al. First observation of a nuclear s-state of a $\Xi$ hypernucleus, ${ }_{\Xi}^{15}$ C. Prog. Theor. Exp. Phys. 2021, DOI: https://doi.org/10.1093/ptep/ptab073 (2021). 073D02, https://academic.oup.com/ptep/article-pdf/2021/7/073D02/ 39257193/ptab073.pdf.

29. Tanaka, H. K. et al. High resolution imaging in the inhomogeneous crust with cosmic-ray muon radiography: The density structure below the volcanic crater floor of Mt. Asama, Japan. Earth Planet. Sci. Lett. 263, 104-113, DOI: https://doi.org/10.1016/j.eps1.2007.09.001 (2007).

30. Morishima, K. et al. Discovery of a big void in khufu's pyramid by observation of cosmic-ray muons. Nature 552, 386-390, DOI: https://doi.org/10.1038/nature24647 (2017).

31. Naka, T. et al. Fine grained nuclear emulsion for higher resolution tracking detector. Nucl. Instruments Methods Phys. Res. Sect. A: Accel. Spectrometers, Detect. Assoc. Equip. 718, 519-521, DOI: https://doi.org/10.1016/j.nima.2012.11.106 (2013).

32. Asada, T., Naka, T., Kuwabara, K.-i. \& Yoshimoto, M. The development of a super-fine-grained nuclear emulsion. Prog. Theor. Exp. Phys. 2017, DOI: https://doi.org/10.1093/ptep/ptx076 (2017).

33. Alexandrov, A. et al. Intrinsic neutron background of nuclear emulsions for directional Dark Matter searches. Astropart. Phys. 80, 16-21, DOI: https://doi.org/10.1016/j.astropartphys.2016.03.003 (2016).

34. Shiraishi, T. et al. Development of a new tracking detector with fine-grained nuclear emulsion for sub-MeV neutron measurement. Prog. Theor. Exp. Phys. 2021, DOI: https://doi.org/10.1093/ptep/ptab030 (2021). 043H01, https://academic. oup.com/ptep/article-pdf/2021/4/043H01/37807850/ptab030.pdf.

35. Naganawa, N. et al. A cold/ultracold neutron detector using fine-grained nuclear emulsion with spatial resolution less than 100 nm. The Eur. Phys. J. C 78, 959, DOI: https://doi.org/10.1140/epjc/s10052-018-6395-7 (2018).

36. Hirota, K. et al. Neutron Imaging Using a Fine-Grained Nuclear Emulsion. J. Imaging 7, DOI: https://doi.org/10.3390/ jimaging7010004 (2021).

37. Hino, M. et al. The ion beam sputtering facility at KURRI: Coatings for advanced neutron optical devices. Nucl. Instruments Methods Phys. Res. Sect. A: Accel. Spectrometers, Detect. Assoc. Equip. 797, 265-270, DOI: https://doi.org/10.1016/j. nima.2015.06.046 (2015).

38. Grünzweig, C., Frei, G., Lehmann, E., Kühne, G. \& David, C. Highly absorbing gadolinium test device to characterize the performance of neutron imaging detector systems. Rev. Sci. Instruments 053708, DOI: https://doi.org/10.1063/1.2736892 (2007).

39. Seki, Y. et al. Experimental Evaluation of Neutron Absorption Grating Fabricated by Oblique Evaporation of Gadolinium for Phase Imaging. Phys. Procedia 88, 217-223, DOI: https://doi.org/10.1016/j.phpro.2017.06.030 (2017).

40. Samoto, T., Takano, H. \& Momose, A. Gadolinium oblique evaporation approach to make large scale neutron absorption gratings for phase imaging. Jpn. J. Appl. Phys. 58, SDDF12, DOI: https://doi.org/10.7567/1347-4065/ab138d (2019).

41. Samoto, T., Takano, H. \& Momose, A. Evaluation of obliquely evaporated gadolinium gratings for neutron interferometry by X-ray microtomography. Mater. Sci. Semicond. Process. 92, 91-95, DOI: https://doi.org/10.1016/j.mssp.2018.05.038 (2019). Material processing of optical devices and their applications.

42. Mishima, K. et al. Design of neutron beamline for fundamental physics at J-PARC BL05. Nucl. Instruments Methods Phys. Res. Sect. A: Accel. Spectrometers, Detect. Assoc. Equip. 600, 342-345, DOI: https://doi.org/10.1016/j.nima.2008.11.087 (2009).

43. Otsu, N. A Threshold Selection Method from Gray-Level Histograms. IEEE Transactions on Syst. Man, Cybern. 9, 62-66, DOI: https://doi.org/10.1109/TSMC.1979.4310076 (1979). 


\section{Acknowledgements}

The neutron experiment at the Materials and Life Science Experimental Facility of the J-PARC was performed under user programs (Proposal No. 2020B0223). The preliminary experiment on the detector was performed at the Research Reactor Institute, Kyoto University (Experiment No. R2138). J. Y. was supported by JSPS KAKENHI Grant Number JP18H05403 (Grant-in-Aid for Scientific Research on Innovative Areas 6005). The authors thank Takenao Shinohara of J-PARC and Yoshichika Seki of Osaka electro-communication University for lending the Siemens star test pattern and the grating, and Atsushi Momose and Tetsuo Samoto of Tohuku University for providing the SEM images shown in figure 3 and for the discussions related to the grating. The authors also thank Atsuhiro Umemoto and Ryuta Kobayashi from Nagoya University for their technical support in using the optical microscope. The authors would like to thank Yukiko Kurakata of the High Energy Nuclear Physics Laboratory at RIKEN for providing administrative support for the entire project.

\section{Author contributions statement}

K.H, T.R.S proposed this study, and T.R.S managed the project. A.M, J.Y, M.H, N.M, N.N produced the neutron detectors. A.M, J.Y, K.H., M.H, G.I, K.M, M.N. performed the experiments. A.M, J.Y, N.N performed observation and scanning of the detectors. A.M, J.Y, A.K performed the analysis. A.M, J.Y, H.E, M.H, K.H, G.I, A.K, M.K, N.M, K.M, J.U.N, M.N, N.N, T.R.S contributed in writing the manuscript. All authors contributed to finalizing the manuscript and approved the final draft. 